Gynäk. Rdsch. 1969;8:I-VIII

\title{
Contents, Vol. 8, 1969
}

Kurzfassungen von Zeitschriftenartikeln

Agarwala, S. N.: Eine nachgehende Studie über die intrauterinen kontrazeptiven

Einlagen (IUCD): Eine indische Erfahrung 174

Anderson, E. E.; Cobb, O. E. und Glenn, J. F.: Die hämorrhagische Cyclophosphamidcystitis 156

Anderson, J.: vide Fisch, R. O.

Apgar, Virginia und Stickle, G.: Angeborene Defekte. Ein Problem des öffentlichen Gesundheitswesens 81

Ashkenazi, H.: vide Toaff, R.

Asnes, R. S.: Atemdepression Neugeborener, bedingt durch Schmerzlinderung bei der Mutter und ihre Behandlung durch Austauschtransfusion 122

A yd, F. J., Jr.: Teenager und Konzeptionsverhütung183

Badib, A. O.; Kurohara, S. S.; Beitia, A. A. und Webster, J. H.: Rezidive nach

Korpuskarzinom. Technik und Ergebnisse der Behandlung 230

Basu, H. K.: Normale Menstruation, Menorrhagien und Fibrinspaltprodukte im

Serum 295

Beard, R. W.: vide Colart, T. M.

Beitia, A. A.: vide Badib, A. O.

Bérard, J.: vide Lefèbre, Y.

Bérard-Bader, M.; Laugier, M.; Loucet, E. und Payan, H.: Die Plazenta bei der akuten kongenitalen Toxoplasmose im Tierversuch 136

Bernard, R. P.; Compton-Nemer, H.; Sakoda, J. M.; Potter, R. G. und Chi,

P. S.: Ergebnisse der Intrauterinpessar-Anwendung in Jugoslawien 170

Birtich, A.: vide Crane, C.

Bolognese, R. J. und Corson, S. L.: Die Konisation der Zervix in der Schwangerschaft 304

Bonilla-Musoles, F.: Elektronenmikroskopische Untersuchungen an der Cervix uteri 197

Bonnar, J.; McNicol, G. P. und Douglas, A. S.: Untersuchung des fibrinolytischen Systems in der Schwangerschaft 291

Bor, S.: vide Morrison, G. D. Borst-Eiler, Els: vide Dudok de Wit, C.

Boy an, C. P.: Der hypovolämische Schock 300

British Cooperative Clinical Group: Gonorrhoe in Grossbritannien 1967 .... 189 British

Cooperative Clinical Group: Primäre und sekundäre Syphilis 1966 - bezogen

auf das Ursprungsland der Patienten 193

Bruce, J.: vide Smith, A. N.

Brux, J. A. de; Dupré-Froment, J. und Mintz, M.: Zytologie der peritonealen

Flüssigkeit, die durch Coelioskopie oder Douglaspunktion gewonnen wurde.

Ihre Bedeutung für die Gynäkologie 28 
Cade, J. F.; Hirsh, J. und Martin, M.: Die Plazentaschranke für Blutgerinnungsfaktoren: Ihre Bedeutung für Blutgerinnungsschwäche bei der Geburt und bei

Blutungen des Neugeborenen 212

Inhaltsverzeichnis III

Callagham, N.: Das Migräne-Syndrom in der Schwangerschaft

20

Canu, M. F.: Frau und Sport 1

Caquet, R.: vide Laroche, $\mathrm{Cl}$.

Cassady, G.: vide Overbach, A. M.

Cheynier, J.-M.: vide Laroche, $\mathrm{Cl}$.

Chi, P. S.: vide Bernard, R. P.

Chow, B. F., et al.\Mütterliche Ernährung während der Schwangerschaft und ihre

Folgen für die Entwicklung der Nachkommen. Eine Studie an Ratten und Men-

schen 98

Chung, C S. und Myrianthopoulos, N. C.: Rassische und pränatale Faktoren bei

grösseren kongenitalen Missbildungen

93

Clark, G. W.: vide Smith, R. E.

Cobb, O. E.: vide Anderson, E. E.

Cobbold, R. J. C.: vide Morrsion, G. D.

Coltart, T. M.; Trickey, N. R. A. und Beard, R. W.: Fetale Blutanalyse als prak-

tisches Hilfsmittel bei der Gefährdung in utero

119

Compton-Nemer, H.: vide Bernard, R. P.

Cooke, B. A.: vide Shirley, J. M.

Corson, S. L.: vide Bolognese, R. J.

Couch, N. P.: vide Crane, C.

Cox, J. E.: vide Morten, D. H.

Crane, C.; Hartsuck, J.; Birtich, A.; Couch, N. P.; Zollinger, R.; Matloff,

J.; Dalen, J. und Dexter, L.: Die Behandlung grösserer Pulmonalembolien . 45 Creveld, S., van

und Schellekens, L. A.: Blutungen in der Pubertät bei Mädchen

mit hämorrhagischer Diathese

299

Dadoune, J. P.: vide Laroche, $\mathrm{Cl}$.

Dalen, J.: vide Crane, C.

Daniel, S. J.: vide Overbach, A. M.

Davies, E.: vide Glow, C.

Delahanty, D. D.: Die chirurgische Korrektur ätiologischer Faktoren uteriner

Erkrankungen bei Stuten 71

Dexter, L.: vide Crane, C.

Doeden, D.: vide Fisch, R. O.

Doll, R. und Vessey, M. P.: Beurteilung seltener Nebenwirkungen von Ovulations-

hemmern 261

Dortmann, A.; Düchting, M. und Küster, F.: Infektionsrisiko der Austausch-

transfusion 115

Douglas, A. S.: vide Bonnar, J.

Douglas, Mary: vide Smith, A. N.

Dowling, E. A.; Gravlee, L. G. und Hutchins, K. E.: Eine neue Technik zur Ent-

deckung des Adenokarzinoms des Endometriums 228

Düchting, M.: vide Dortmann, A. 
Dudok de Wit, C. und Borst-Eiler, Els : Misserfolg in Verwendung von Anti-DImmunoglobulin zur Prophylaxe der Anti-Rhesus-Immunisation nach massiver feto-maternaler Hämorrhagie. Bericht über 4 Fälle 133

Dupré-Froment, J.: vide Brux, J. A. de

Eckstein, P.: Zum Wirkungsmechanismus der Intrauterinpessare 249

IV

Inhaltsverzeichnis

Espenschade, A. S.: Die Bedeutung von körperlicher Betätigung bei gesunden

Frauen im Alter von 35 bis 80 Jahren 243

Etheridge, M. J.: Herzkrankheit und Schwangerschaft 214

Farman, R. S.: Sectio caesarea bei der Stute 74

Fassett, J. D.: vide Gordis, L.

Finkelstein, R.: vide Gordis, L.

Fisch, R. O.; Doeden, D.; Lansky, L. und Anderson, J.: Mütterliche Phenyl-

ketonurie $\quad 308$

Forest, M.: vide Laroche, $\mathrm{Cl}$.

Foster, D. N.: vide Morrison, G. D.

Frater, R.: vide Wohlgemut, G.

Gaffney, T. J.: vide Jacobi, J. M.

Galian, Ph. : vide Laroche, $\mathrm{Cl}$.

Gillivray, Mac; Rose, G. A. und Rowe, B,: Blutdruckmessungen in der Schwan gerschaft 265

Ginther, O. J.: Der Einfluss des «Intrauterine-Device» auf die Ovarialfunktion beim Schaf 182

Glenn, J. F.: vide Anderson, E. E.

Glow, C.; Sriver, C. R. und Davies, E.: Resultate eines Massen-Screening auf Hyperaminoacidämie bei neugeborenen Kindern 22

Gordis, L.; Fassett, J. D.; Finkelstein, R. und Tayback, M.: Schwangerschaft

bei Jugendlichen: Ein Verhütungsprogramm unter Krankenhausleitung ... 15

Gravlee, L. G.: vide Dowling, E. A.

Grégoire, J.: vide Laroche, Cl.

Griffin, N. B.: Veränderungen im Bereich der Scheide nach Strahlentherapie . . 36

Gruchy, C. G. de : vide O’Sullivan, E. F.

Hall, W. L.; Sobel, A. I.; Jones, C. P. und Parker, R. T.: Postoperative Infektion

der Beckenorgane durch Anaerobier 232

Harrison, C. P.: 1st Interruptio die Lösung für schwangere Teenager? 185

Harrison, M. J. G.: vide Lewis, P. D.

Hartstuck, J.: vide Crane, C.

Herzberg, M.: vide Toaff, R.

Hirch, J.: vide O’Sullivan, E. F.

Hirsh, J.: vide Cade, J. F.

Hirt, H. R.: Beitrag zur Symptomatologie der zentralnervösen Residualschäden

durch Icterus gravis neonatorum 128

Hirvensalo, M.: vide Yao, Alice C.

Hutchins, K. E.: vide Dowling, E. A. 
Iizuka, R.; Sawada, Y.; Nishina, N. und Ohi, M.: Die psychosomatische Entwicklung von Kindern nach künstlicher Befruchtung 131

Itelson, I.: vide Kaori, $\mathrm{M}$.

Jacobi, J. M.; Powell, L. W. und Gaffney, T. J.: Immunochemische Vermehrung von menschlichem Transferrin bei Schwangerschaft und während der Einnahme von Ovulationshemmern 167

Jacobs, E. M.: vide Johnson, F. D.

James, H.: Der Einfluss mütterlicher Stressituationen auf den Feten272

Inhaltsverzeichnis $\mathrm{V}$

Jarrahi-Zadeh, A., et al.: Emotionalität und kognitive Regungen im Wandel von

Schwangerschaft und frühem Wochenbett 147

Johnson, F. D.; Jacobs, E. M. und Silliphant, W. M.: Trophoblastische Tumoren

des Uterus. Probleme der Methotrexattherapie 224

Jones, C. P.: vide Hall, W. L.

Kaori, M. und Itelson, I.: Behandlung der Thyreotoxikose in der Schwangerschaft 149

Kilpatrick, N.: vide Morrison, J.

Kurohara, S. S.: vide Badib, A. O.

Küster, F.: vide Dortmann, A.

Laing, J. A.: Studien über Fertilität und Infertilität bei Rindern, Schafen und Schwei-

nen $\quad 75$

Lamquin, R.: vide Lefèbre, $Y$.

Lansky, L.: vide Fisch, R. O.

Laroche, Cl. ; Grégoire, J.; Caquet, R.; Modigliani, R.; Galian, Ph. ; Forest, M.; Dadoune, J. P. und Cheynier, J.-M.: Akute Lebersteatose in der Schwangerschaft (Seehan-Syndrom). Studie über Histologie und Ultrastruktur .... 306

Laufenstein-Duffy, H.: Indikationen und Technik der Curettage bei Stuten ... 69

Laugier, M.: vide Bérard-Bader, $M$.

Lauritzen, Ch. : Gonadotropine in der Präpubertät und Pubertät 194

Lefèbre, Y.; Moutquin, J.-M.; Lamquin, R. und Bérard, J.: Die Uterusperfora-

tionen mit Intrauterinpessaren177

Lewis, P. D. und Harrison, M. J. D.: Unwillkürliche Bewegungen bei Patienten

unter oralen Kontrazeptiva 165

Lewit, S.: vide Tietze, C.

Lind, J.: vide Yao, Alice C.

Lind, J. F., et al.: Sodbrennen in der Schwangerschaft: Manometrische Untersu-

chungen 143

Long, N. T.: vide Minh, H. N.

Lorincz, A. B.: Die gynäkologische Betreuung des heranwachsenden Mädchens 10

Loucet, E.: vide Bérard-Bader, M.

Lyons, D. J.: Entwicklung eines Programmes für schwangere Teenager durch Zu-

sammenarbeit zwischen Schule, Gesundheitsamt und Bundesbehörden .... 187

Markush, R. E. und Seigel, D. G.: Hormonelle Kontrazeptiva und Trend der

Thromboembolie-Mortalität in den Vereinigten Staaten 252

Martin, M.: vide Cade, J. F.

Matloff, J.: vide Crane, C.

Matsumato, S.: vide Sato, T. 
McCarthy, R. A.: vide O’Sullivan, E. F.

McLean, N.: vide Smith, A. N.

McNicol, G. P.: vide Bonnar, J.

Merkatz, I. R.; New, M. J.; Peterson, R. E. und Seaman, M. P.: Pränatale Dia gnose des Adrenogenitalen Syndroms mittels der Amniozentese 313

Meyer, W.: Klinik, Pathogenese und Therapie des Schockzustands bei Sepsis durch gramnegative Erreger 62

Michel-Wolfromm, Hélène: Psychosomatische Störungen in der Menopause . . 63

Minh, H. N.; Long, N. T. und Que, N. T.: Spontane Uterusruptur in der ersten

Schwangerschaftshälfte $\quad 146$

VI

Inhaltsverzeichnis

Mintz, M.: vide Brux, J. A. de

Modigliani, R.: vide Laroche, $\mathrm{Cl}$.

Morrison, G. D.; Cobbold, R. J. C; Bor, S.; Spitzer, R. J.; Foster, D. N. und

Willcox, R. R.: Behandlung der gonorrhoischen Urethritis 191

Morrison, J. und Kilpatrick, N.: Niedrige Oestriolausscheidung im Harn von

Schwangeren unter Prednison-Therapie

19

Morten, D. H. und Cox, J. E.: Die erschwerte Geburt bei Kühen 152

Moutquin, J.-M.: vide Lefèbre, Y. Myrianthopoulos, N. C.: vide Chung, C. S.

Nadler, H. L.: Entdeckung von erblichen Fehlanlagen vor der Geburt 87

Nadler, H. L.: Entdeckung von fehlerhaften Erbanlagen vor der Geburt .... 89

Napp, E. E.: vide Turkel, W. V.

New, M. J.: vide Merkatz, I. R.

Nishina, N.: vide Iizuka, R.

Norris, H. J. und Taylor, H. B.: Prognose der Granulosa-Thekazelltumoren des

Ovars (mit Kommentar) 25

Odell, G. B.: vide Weldon, V. V.

Ohi, M.: vide Iizuka, R.

O’Sullivan, E. F.; Hirch, J.; McCarthy, R. A. und Gruchy, C. G. de: Heparin

in der Behandlung des Morbus thrombo-embolicus: Verabreichung, Kontrolle

und Resultate 42

Overbach, A. M.; Daniel, S. J. und Cassady, G.: Der Wert histologischer

Schnittuntersuchungen der Nabelschnur in der Behandlung potentieller perinataler Infektionen 311

Panigel, M.: Die Physiologie der plazentaren Zirkulation beim Menschen und anderen Primaten 100

Parker, R. T.: vide Hall, W. L.

Payan, H.: vide Bérard-Bader, N.

Payer, J.; Stojkovic, J. und Rudolf, V.: Die geburtstraumatische Nierenruptur

beim Neugeborenen 124

Peterson, R. E.: vide Merkatz, I. R.

Poller, L.; Thomson, Jean M.; Taiowo, A. und Priest, C. M.: Blutgerinnung bei oraler Kontrazeption durch ein Progesteron-Präparat259

Potter, R. G.: vide Bernard, R. P.

Powell, L. W.: vide Jacobi, J. M. 
Priest, C. M.: vide Poller, L.

Quan, S. H.: «Factitial Proctitis» Strahlenschäden des Rectums nach Bestrahlung der Karzinome der Cervix uteri 38

Que, N. T.: vide Minh, H. N.

Reynolds, L. M.: vide Smith, R. E.

Rose, G. A.: vide Gillivray, Mac

Rothauge, C. F. und Ruile, K.: Die operative Behandlung des geschädigten

Harnleiters nach gynäkologischer Karzinomtherapie 46

Rowe, B.: vide Gillivray, Mac Ruckley, C. V.: vide Smith, A. N. Rudolf, V.: vide Payer, J.

Ruile, K.: vide Rothauge, C. F.

Inhaltsverzeichnis VII

Sager, F. C.: Vorgehen und medikamentöse Therapie von Uteruserkrankungen bei

Stuten 67

Sakoda, J. M.: vide Bernard, R. P.

Sato, T.; Shinada, T. und Matsumato, S.: Klinische und biochemische Unter-

suchungen an einem Fall mit virilisierendem Arrhenoblastom

27

Sawada, Y.: vide Iizuka, $\mathrm{R}$.

Schellekens, L. A.: vide Creveld, S., van

Schneeweiss, B. und Wernitz, C.: Der Einfluss der kindlichen Blutgruppe ABO

auf den transplazentaren Übertritt fetaler Erythrozyten 112

Schwartz, A.: vide Toaff, R.

Scott, R. B.: Ein Überblick über Todesfälle und ernstliche Erkrankungen im

Zusammenhang mit dem Gebrauch von Intrauterinpessaren 180

Seaman, M. P.: vide Merkatz, I. R.

Seigel, D. G.: vide Markush, R. E.

Shinada, T.: vide Sato, T.

Shirley, J. M. und Cooke, B. A.: Stoffwechsel von Dehydroepiandrosteron in den getrennten Nebennierenrindenschichten von menschlichen Feten und von Neugeborenen 23

Silliphant, W. M.: vide Johnson, F. D.

Silverman, W. A.: Diagnose und Behandlung der retrolentalen Fibroplasie nach

Sauerstoffgaben 126

Smith, A. N.; Douglas, Mary; McLean, N.; Ruckley, C. V. und Bruce, J.:

Intestinale Komplikationen nach Beckenbestrahlung wegen gynäkologischer

Karzinome (mit Kommentar) 40

Smith, R. E.; Reynolds, I. M. und Clark, G. W.: Experimentelle Listeriose.

I. Inokulation bei tragenden Schafen 138

Sobel, A. I.: vide Hall, W. L.

Spitzer, R. J.: vide Morrison, G. D.

Sriver, C. R.: vide Glow, C.

Sterman, M. B.: Beziehung zwischen intrauteriner Aktivität und mütterlichem

Schlaf 141

Stickle, G.: vide Apgar, Virginia

Stojkovic, J.: vide Payer, J.

Stone, M. L.: vide Turkel, W. V.

Tabiowo, A.: vide Poller, L. 
Tayback, M.: vide Gordis, L.

Taylor, H. B.: vide Norris, H. J.

Thomson, Jean M.: vide Poller, L.

Thrombose und Embolie im Bereich der Geburtshilfe und Gynäkologie (Kommentar) 208

Thurstone, Ph. B.: Die therapeutische Schwangerschaftsunterbrechung 245

Tietze, C. und Lewit, S.: Schwangerschaftsunterbrechung 241

Tietze, Ch. : Orale und intrauterine Kontrazeption. Wirksamkeit und Sicherheit 161 Toaff, R.;

Ashkenazi, H.; Schwartz, A. und Herzberg, M.: Der Einfluss von

Oestrogenen und Gestagenen auf die Milchzusammensetzung von Wöchnerinnen 24 Trickey, N.

R. A.: vide Colart, T. M. Turkel, W. V.; Stone, M. L. und Napp, E. E.: Ein geriatrisch-

gynäkologischer

Überblick 253

VIII

Inhaltsverzeichnis

Vessey, M. P.: vide Doll, R.

Watteville, H., de: Therapeutische Schwangerschaftsunterbrechung durch Injek-

tion von $10 \%$ Kochsalzlösung in die Amnionhöhle 247

Webster, J. H.: vide Badib, A. O.

Weldon, V. V. und Odell, G. B.: Mortalitätsrisiko der Austauschtransfusion 117

Wernitz, C.: vide Schneeweiss, C.

Willcox, R. R.: vide Morrison, G. D.

Wist, A.: vide Yao, Alice C.

Wohlgemut, G. und Frater, R.: Spätfolgen der Schwangerschaft bei Frühgeborenen 221

Yao, Alice C.; Hirvensalo, M. und Lind, J.: Plazentare Transfusionsrate und uterine Kontraktionen 108

Yao, Alice C.; Wist, A. und Lind, J.: Das Blutvolumen des durch Sectio Caesarea

entbundenen Neugeborenen 111

Zollinger, R.: vide Crane, C.

Kasuistik

Felder, K. D.: Beitrag zur Frage der Genitalblutungen bei hämorrhagischen Dia-

thesen 315

Prüfungsfragen für Fachärzte

Prüfungsfragen 78, 158, 238, 319

Richtige Antworten der Fragen Nr. 1-10 aus Heft Nr.l 160

Richtige Antworten der Fragen Nr. 11-20 aus Heft Nr. $2 \quad 240$

Richtige Antworten der Fragen Nr. 21-30 aus Heft Nr. 3320

Inhaltsverzeichnis von Band $8 \quad$ nach 320

S. Karger AG, Arnold-Böcklin-Strasse 25, 4000 Basel 11 (Schweiz)

Alle Rechte, insbesondere das der Übersetzung in andere Sprachen, vorbehalten.

Ohne ausdrückliche Genehmigung des Verlages ist es auch nicht gestattet,

diesen Band oder Teile daraus auf photomechanischem Wege (Photokopie, Mikrokopie) zu

vervielfältigen.

(C)

Copyright 1969 by S. Karger AG, Basel

Printed in Switzerland by Buchdruckereí Natíonal-Zeitung, Basel 
Klischees: Steiner \& Co. AG, Basel 